\title{
Atuação discente e docente da área da saúde no CHC-UFPR para o enfrentamento da pandemia COVID-19 em 2020
}

\author{
RESUMO
}

Leonelo Dell Anhol Almeida leoneloalmeida@utfpr.edu.br Universidade Tecnológica Federal do Paraná, Curitiba, Paraná

Thais De Oliveira Queiroz thaisqueiroz@alunos.utfpr.edu.br Universidade Federal do Paraná, Curitiba, Paraná
Este artigo relata a contribuição dos e das docentes e discentes da área da saúde do Complexo Hospital de Clínicas da Universidade Federal do Paraná (CHC/UFPR) em colaboração com o Sistema Único de Saúde (SUS). Trata de descrever a atuação dessas pessoas na instituição por meio da análise de dados fornecidos e divulgados pelo hospital escola no período de março a julho do ano de 2020. As contribuições estão relacionadas ao apoio administrativo, orientação telefônica e/ou online à população, atendimentos, entre outras, sob supervisão direta dos profissionais da saúde do CHC/UFPR. Neste sentido, o registro histórico tem como objetivo exemplificar a relevância das Universidades Públicas no Brasil ao destacar a importância dos papéis docente e discente universitários para o enfrentamento de diversas demandas sociais.

PALAVRAS-CHAVE: Universidade pública. Saúde pública. Ciências da saúde. COVID-19. Complexo Hospital de Clínicas da UFPR. 


\section{INTRODUÇÃO}

A COVID-19 foi notificada pela primeira vez em 31 de dezembro de 2019 na China, e trata-se de um novo agente do coronavírus que causa infecção respiratória, em casos leves. Os sintomas são os mesmos de um resfriado comum, no entanto, podem evoluir para quadro que necessite de internação hospitalar e cuidados de terapia intensiva. Segundo o Ministério da Saúde, o primeiro caso confirmado no Brasil foi registrado em 26 de fevereiro de 2020 e até dia 29 de junho de 2020 foram confirmados 2.552.265 casos, sendo que destes 90.134 evoluíram para óbito.

Este relato tem o objetivo de registrar a atuação da Universidade em questão, descrevendo como os estudantes supervisionados por seus discentes têm reforçado o atendimento no Sistema Único de Saúde (SUS) e como tem contribuído para a minimização dos efeitos da pandemia.

Segundo o boletim diário divulgado pela Secretaria Estadual de Saúde do Paraná (SESA, 2020), desde o primeiro caso notificado no Estado em 12/03/2020 até o dia 29/07/2020, já foram confirmados 70.155 casos da COVID-19, sendo que deste total 1.792 foram a óbito, 1.273 estão internados, sendo 538 em unidade de terapia intensiva e 735 em leitos clínicos.

O Hospital de Clínicas da UFPR disponibilizou boletim oficial dos casos suspeitos e confirmados atendidos na instituição (Complexo Hospital de Clínicas da Universidade Federal do Paraná, 2020). O último boletim eletrônico do hospital que foi divulgado até a data desta pesquisa se deu no dia 28/07/2020 e apontou que foram atendidos 359 casos confirmados de COVID-19 na instituição, sendo que destes 55 foram a óbito e 235 receberam alta. Estando internados na unidade de terapia intensiva 44 pacientes com casos confirmados e outros 13 que em investigação, permanecem internados em enfermaria 44 pacientes com casos confirmados e outros 9 casos em investigação.

Dados do Infogripe, da Fundação Oswado Cruz (FIOCRUZ), apontaram que no Paraná entre os meses de março e abril, em comparação ao ano anterior o Estado apresentava um número três vezes maior de internações por Síndrome Respiratória Aguda Grave (SRAG), tal aumento apontou a possibilidade de subnotificações dos casos da COVID-19 no Estado, fato que levou as autoridades de saúde a readequarem os serviços para suprir as necessidades hospitalares. Diante do exposto, as universidades proveram recursos para as mais diversas demandas para que o sistema de saúde entrasse em colapso.

\section{MÉTODO}

O levantamento de dados para esta nota técnica se deu por meio de pesquisa documental, que utilizou materiais cedidos e divulgados pelo hospital e por pesquisas em sites das três esferas de governo. Trata-se da discussão e da análise da experiência de formação e atuação na pandemia da COVID-19. Para isto foram identificadas as áreas de formação e a atuação dos discentes no contexto da 
pandemia, entre o período de março a julho de 2020, nas dependências do Complexo Hospitalar da Universidade Federal do Paraná.

\section{DESENVOLVIMENTO}

Embora as universidades públicas brasileiras atuem no campo de pesquisa, produzindo quase toda a totalidade de pesquisas nacionais e serem campos de formação de excelentes profissionais, um informativo técnico elaborado pela Câmara dos Deputados (Câmara dos Deputados, 2020), aponta que entre os anos de 2014 e 2018 no Brasil, os investimentos em educação superior sofreram uma redução de 15\%: de $\mathrm{R} \$ 39,2$ bilhões para 33,4 bilhões. A pesquisa foi realizada com base nos investimentos efetivamente realizados no período, através de dados coletados no SIAFI (Sistema Integrado de Administração Financeira do Governo Federal) e posteriormente corrigidos pelo Índice Nacional de Preços ao Consumidor Amplo (IPCA).

Além disso, a pesquisa Percepção Pública da Ciência e Tecnologia no Brasil, publicada em 2019 pelo Centro de Gestão e Estudos Estratégicos (CGEE) e pelo Ministério da Ciência, Tecnologia, Inovações e Comunicações (MCTIC), retrata que as universidades públicas são responsáveis pela maior parte da produção científica nacional, conforme dados da base Web of Science, compilados pela Clarivate Analytics entre 2014 e 2018. As pesquisas apontam que dentre as 50 instituições que mais produzem trabalhos científicos no Brasil, 44 são universidades públicas. Essa pesquisa ainda entrevistou 2.200 pessoas com idade superior a 16 anos e verificou que ainda não há um reconhecimento desta produção pela sociedade, pois "poucos entrevistados souberam citar o nome de um cientista ou instituição de ciências; $90 \%$ dos brasileiros não se lembram ou não sabem apontar um cientista do País; $88 \%$ não se lembram ou não sabem indicar instituição do setor. Nem mesmo as universidades foram muito citadas, embora sejam os principais centros de produção de conhecimento científico" (Centro de Gestão e Estudos Estratégicos, 2019). Tais resultados são preocupantes e retratam a urgência em desenvolver métodos de divulgação para a sociedade sobre trabalhos realizados pelas universidades públicas no país.

No Brasil, por meio do Ministério da Saúde, estudantes da área da saúde foram chamados para atuarem no enfrentamento da COVID-19 no Sistema Único de Saúde (SUS). A exemplo disto, o Hospital de Clínicas da Universidade Federal do Paraná adotou estratégias para suprir uma possível carência de recursos humanos em suas dependências. Sendo assim, uma das ações adotadas foi realizar um chamamento para cadastro de estudantes da área da saúde da própria universidade para realização de trabalho voluntário nas dependências da instituição.

Os e as discentes realizaram o cadastro por meio de um formulário próprio, no qual declaram estar em plena condição de saúde e não pertencer a nenhum grupo de risco, conforme estabelecido pelo Ministério da Saúde. Cofessam ciência de todos os riscos envolvidos e se comprometem a cumprir todas as regras da instituição e do Ministério da Saúde em relação à prevenção de transmissão à comunidade, devendo utilizar todos os equipamentos de proteção indicados conforme o grau de risco a cada setor de exposição. 
Segundo a coordenação do programa, 357 estudantes se cadastraram, sendo 10 do curso de Biomedicina, 44 de Terapia Ocupacional, 32 de Enfermagem, 38 de Farmácia, 18 de Fisioterapia, 145 de Medicina, 18 de Nutrição, 36 de Odontologia, 15 de Psicologia e um de Serviço Social. As atividades serão acompanhadas por profissionais da área da saúde com registro nos respectivos conselhos profissionais competentes, bem como sob orientação docente, e ao final da contribuição a universidade fornecerá um certificado de participação, contendo a descrição das atividades realizadas e a carga horária.

O hospital também contou com o apoio dos 501 estudantes residentes médicos e multiprofissionais regularmente matriculados em atividade no $\mathrm{CHC}$ dos cursos de Enfermagem, Fisioterapia, Nutrição, Farmácia, Farmácia-bioquímica, Serviço Social, Psicologia, Odontologia, Terapia Ocupacional e Medicina, visto que, atendimentos ambulatoriais e cirurgias eletivas estavam suspensas no período.

Neste sentido, considerando as necessidades advindas da COVID-19, a Gerência de Ensino e Pesquisa (GEP), por meio do Setor de Gestão do Ensino, da Comissão de Residência Médica (COREME) e da Comissão de Residência Multiprofissional (PRIMAH/COREMU), em parceria com outras áreas do CHC, realizou capacitação e treinamentos a estes estudantes.

Os residentes do curso de Psicologia realizaram atendimentos aos familiares de pacientes com COVID-19, por via digital, além disto, também prestaram apoio psicológico aos funcionários do $\mathrm{CHC}$ e aos residentes dos demais programas; os residentes de Enfermagem também tiveram importante papel na organização e realização da vacinação contra a gripe aos funcionários do hospital. Além de estarem capacitados para atender outras demandas relacionadas a assistência de enfermagem aos pacientes internados; os residentes de medicina seguiram realizando triagem de todos os pacientes que necessitaram acessar a área interna do hospital. Assim, quando eram identificados sinais e sintomas para COVID-19 seguia-se o encaminhamento para um consultório específico para dar sequência ao atendimento por suspeita de COVID-19. Outros discentes prestaram apoio administrativo realizando ligações para remanejamento de consultas e cirurgias eletivas e orientação quanto às dúvidas dos pacientes. Adicionalmente, 28 residentes da Medicina Veterinária atuaram em áreas administrativas do Hospital em apoio a diversas atividades gerenciais.

\section{CONSIDERAÇÕES FINAIS}

Esta nota apresentou uma estratégia adotada pelos gestores da saúde de um hospital público, que considerou o recrutamento de discentes de uma universidade pública para o atendimento das demandas do Sistema Único de Saúde de maneira gratuita e com qualidade à sociedade.

De acordo o art. 43 da lei n. 9.394, de 20 de dezembro de 1996 (Brasil, 1996), que estabelece as diretrizes e bases da educação nacional, uma das finalidades do ensino superior está relacionada a "VI - estimular o conhecimento dos problemas do mundo presente, em particular os nacionais e regionais, prestar serviços especializados à comunidade e estabelecer com esta uma relação de reciprocidade". O caso da pandemia nos mostra que as demandas de ensino, neste caso, se adequaram às necessidades atuais, tanto na questão educacional quanto na prestação de serviços à comunidade. 
Neste sentido, ressaltamos que o Sistema Único de Saúde e as Universidades Públicas, mesmo sofrendo cortes de investimentos e ataques políticos, seguiram protagonizando ações para o enfrentamento da pandemia, mostrando que o investimento governamental em educação, ciência e tecnologia é devolvido à sociedade através de pesquisas e contribuições das mais diversas possíveis. Ressaltamos que a atuação dos e das discentes e docentes, neste caso mostrou-se de extrema relevância para manter o controle e a organização no serviço hospitalar no período relatado. 


\title{
Students and professors activities in the health area at CHC-UFPR to face the COVID- 19 pandemic in 2020
}

\begin{abstract}
This article reports on the contribution of health professors and students of the Hospital de Clínicas Complex of the Federal University of Paraná (CHC/UFPR) in collaboration with the Unified Health System (SUS). It aims to describe the performance of these people in the institution through the analysis of data provided and disseminated by the teaching hospital from March to July of the year 2020. The contributions go from administrative support, telephone and/or online guidance to the population, assistance, among others, to direct supervision of CHC/UFPR health professionals. In this sense, the historical record aims to exemplify the relevance of Public Universities in Brazil by highlighting the importance of university teaching and student roles in facing various social demands.
\end{abstract}

KEYWORDS: Public university. Public health. Health Sciences. COVID-19. UFPR Hospital de Clínicas Complex. 


\section{AGRADECIMENTOS}

Agradecemos aos professores, Dr. Jorge Vinícius Cestari Félix (Chefe do Setor de Gestão de Ensino do Complexo Hospital de Clínicas da UFPR), Dr. Rosires Pereira de Andrade (Gerente de Ensino e Pesquisa do Complexo Hospital de Clínicas da UFPR) e a Me. Graciele de Matia (Chefe da Unidade de Pesquisa Clínica do Complexo Hospital de Clínicas da UFPR), pela autorização e colaboração.

\section{REFERÊNCIAS}

Brasil. Lei de Diretrizes e Bases da Educação Nacional. 1996. Disponível em: http://www.planalto.gov.br/ccivil 03/Leis/L9394.htm Consulta em 11/04/2020.

Brasil. Portaria no 356, de 20 de março de 2020. Dispõe sobre a atuação dos alunos dos cursos da área de saúde no combate à pandemia do COVID-19 (coronavírus). Disponível

em: http://www.planalto.gov.br/CCIVIL 03/Portaria/PRT/Portaria\%20n\%C2\%BA\%203 56-20-mec.htm Consulta em 07/05/2020.

Brasil. Portaria no 492, de 23 de março de 2020. Institui a Ação Estratégica "O Brasil Conta Comigo", voltada aos alunos dos cursos da área de saúde, para o enfrentamento à pandemia do coronavírus (COVID-19). Disponível em: http://www.in.gov.br/en/web/dou/-/portaria-n-492-de-23-de-marco-de-202024931744 Consulta em 25/04/2020.

Brasil. Portaria no 580, de $\mathbf{2 7}$ de março de 2020. Dispõe sobre a Ação Estratégica "O Brasil Conta Comigo - Residentes na área de Saúde", para o enfrentamento à pandemia do coronavírus (COVID-19). Disponível em: http://www.in.gov.br/en/web/dou/-/portaria-n-580-de-27-de-marco-de-2020250191376 Consulta em 25/04/2020.

Câmara dos Deputados. Ministério da educação: despesas primárias pagas 20142018 e impacto da ec no 95/2016 (teto de gastos). Informativo Técnico no 6/2019conof/cd. https://www2.camara.leg.br/orcamento-da-uniao/estudos/2019/inf 62019-ministerio-educacao-despesas-primarias-pagas Consulta em 13/04/2020.

Centro de Gestão e Estudos Estratégicos (CGEE). Percepção pública da C\&T no Brasil - 2019. Disponível em: https://www.cgee.org.br/documents/10195/734063/CGEE resumoexecutivo Pe rcepcao pub CT.pdf Consulta em 13/04/2020. 
Complexo Hospital de Clínicas da Universidade Federal do Paraná. BOLETIM CHCUFPR/COVID19. Disponível em: Intranet/Boletim Epidemiológico 89 CHC-COVID19. Consulta em 30/07/2020.

Complexo Hospital de Clínicas da Universidade Federal do Paraná. Estudantes da área da saúde poderão ser voluntários. Disponível em : http://www2.ebserh.gov.br/web/chc-ufpr/noticia-aberta/-

Lasset publisher/Zo21hrThpSTk/content/id/5068289/2020-03-edital-COVID-19trabalho-voluntario-no-chc Consulta em 02/05/2020.

Fundação Oswaldo Cruz. Infogripe. Monitoramento dos casos reportados de síndrome respiratória aguda grave (SRAG) hospitalizados. Disponível em: http://info.gripe.fiocruz.br Consulta em 25/04/2020.

Ministério da saúde. Coronavírus. Disponível em: https://coronavirus.saude.gov.br/ Consulta em 11/04/2020.

Ministério da saúde. COVID-19 NO BRASIL . Disponível em: https://susanalitico.saude.gov.br/extensions/COVID-19 html/COVID$19 \mathrm{html.html}$ Consulta em 30/07/2020.

Secretaria de Estado da Saúde do Paraná - SESA. Boletim - Informe Epidemiológico Coronavírus (COVID-19). Disponível em: http://www.saude.pr.gov.br/Pagina/Coronavirus-COVID-19 Consulta em 30/07/2020.

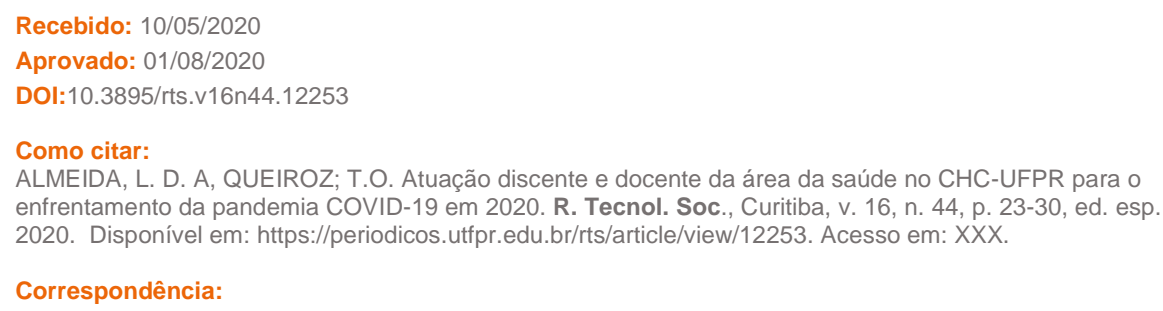

Direito autoral: Este artigo está licenciado sob os termos da Licença Creative Commons-Atribuição 4.0 Internacional.

\section{(c) (1)}

